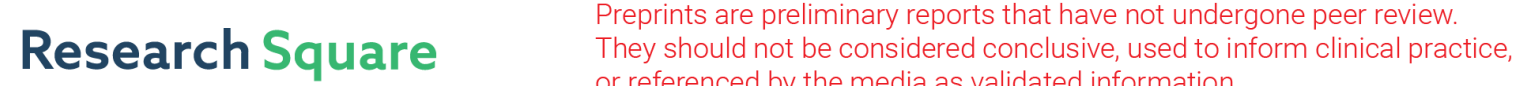 or referenced by the media as validated information. \\ Comprehensive Analysis of Clinical Prognosis and Molecular Characteristics of LTB4R2 in Clear Cell Renal Cell Carcinoma
}

\section{Xia Yuan}

Hunan Cancer Hospital

Chenhui Luo

Hunan Cancer Hospital

Yi He

Hunan Cancer Hospital

Wei Wang ( $\nabla$ wangweizzly@sina.com )

Hunan Cancer Hospital

\section{Research Article}

Keywords: BLT2 expression, Clear cell renal cell carcinoma, Prognosis, Immune infiltration

Posted Date: July 26th, 2021

DOI: https://doi.org/10.21203/rs.3.rs-632886/v1

License: (c) (i) This work is licensed under a Creative Commons Attribution 4.0 International License. Read Full License 


\section{Abstract}

\section{Background}

Clear cell renal cell carcinoma (ccRCC) is the most common renal cancer. LTB4 receptor 2 (LTB4R2, also called BLT2), as a chemokine receptor, has been reported to be upregulated in different tumors and associated with tumor cell proliferation and invasion. However, the correlation between BLT2 expression and its prognostic value in ccRCC remain to be explored.

\section{Methods}

We used the TCGA and GEO databases to evaluate the association between BLT2 expression and clinical outcome of ccRCC. The top 300 genes that most positively correlated with BLT2 were selected for functional enrichment analysis. Correlations between BLT2 expression and tumor immune characteristics in cCRCC were evaluated based on TIMER2.0.

\section{Results}

BLT2 expression was higher in ccRCC than normal tissues. Kaplan-Meier survival analysis indicated that high BLT2 expression was significantly correlated with poor overall survival ( $H R=1.75, p \otimes 0.001)$ and disease-specific survival $(H R=1.60, p \otimes 0.014)$ for patients with ccRCC. In addition, the findings revealed that M1 marker genes had no significant correlations with BLT2 expression in CCRCC, while moderate correlations were observed between BLT2 expression and M2 marker genes. The marker genes of Tregs and T cell exhaustion positively correlated with BLT2 expression in cCRCC (p『0.001).

\section{Conclusion}

BLT2 may serve as a novel prognostic biomarker and correlate with tumor immune microenvironment shaping in cCRCC. BLT2 expression potentially contributes to the regulation of TAMs, T cell exhaustion, and Tregs activation in ccRCC, providing new approaches to drive the development of new immunotherapeutic strategies for ccRCC.

\section{Introduction}

Clear cell renal cell carcinoma (ccRCC) is the most common renal cancer, accounting for more than $80 \%$ of all diagnoses ${ }^{[1,2]}$. The primary defining feature of CCRCC are consistent metabolic abnormalities featuring highly elevated lipid and glycogen deposition. ccRCC is frequently associated with broadly inactivated von Hippel-Lindau tumor suppressor ( $\mathrm{pVHL}$ ) function whose tumour suppressor activity is dependent on its downregulation of hypoxia pathways ${ }^{[3,4]}$. cCRCC is a heterogeneous and lethal malignancy with a poor prognosis. Surgical resection is currently the primary treatment for localized cCRCC, however, ccRCC is frequently diagnosed at an advanced stage and prone to recur ${ }^{[5]}$, and develop unpredictable metastases even with proper treatment ${ }^{[6]}$. The number of targeted pathways in ccRCC 
therapy was limited, thus no therapeutic options were available for non-responding patients ${ }^{[7]}$. The prognosis for patients with metastatic CCRCC is poor of which only $8 \%$ to $11.7 \%$ survive 5 years ${ }^{[8]}$. Therefore, it is urgent to explore novel candidate genes that serve as prognostic biomarkers or novel potential targets for $\mathrm{CCRCC}$ treatment.

There is evidence that unwanted inflammation could affect cancer development and progression. Therefore a growing body of studies deconstruct the roles and outline underlying mechanisms of inflammation in tumors. Gene expression analyses revealed that ccRCC interacted with a particularly inflamed tumor microenvironment compared to other tumor types ${ }^{[9]}$. It was reported that proinflammatory cytokine IL-1 $\beta$ contributed to a pro-tumorigenic microenvironment in ccRCC ${ }^{[10]}$ and chronic inflammation is associated with a poor prognosis and a limited response to sunitinib for CcRCC ${ }^{[11]}$. Given these significant effects of the inflammatory milieu on the prognosis of patients with ccRCC, interrogation of the predictive power and molecular immune characteristics of inflammatory biomarkers in ccRCC prognosis therapy is warranted ${ }^{[12]}$.

LTB4 is a bioactive lipid cytokine synthesized from arachidonic acid by the sequential action of 5lipoxygenase (5-LO) and leukotriene A4 hydrolase ${ }^{[13]}$. LTB4 is an important early mediator of neutrophilic inflammation through high-affinity G-protein coupled receptor BLT1, resulting in chemotaxis of inflammatory cells dominated by neutrophils and thus instigating pathological inflammation in a multitude of chronic diseases including cancer ${ }^{[14,15]}$. The LTB4 receptor 2 (LTB4R2, also called BLT2) shares $45 \%$ homology with BLT1 at the amino acid level and BLT2 was less abundant compared to BLT1 [16]. However, BLT2 is more ubiquitously expressed on the surface of various cell types and transduces signals in response to multiple eicosanoids ${ }^{[17]}$.

BLT2 is upregulated in a variety of human cancers and several in vitro studies explored the roles of BLT2 receptor signaling in different cancer cells ${ }^{[18]}$. It has been demonstrated that BLT2 is a pro-tumorigenic mediator during cancer progression and BLT2 blockade significantly attenuates Ras-induced transformation, suggesting that BLT2 may serve as a therapeutic target for anti-cancer drug development [19]. BLT2 confers invasiveness in aggressive breast cancer cells by regulating IL-8 ${ }^{[19]}$. Furthermore, it was found that the expression of BLT2 was considerably elevated in the multidrug-resistant MCF-7/DOX cells where BLT2 played a principal role in mediating paclitaxel resistance ${ }^{[20]}$. In addition, overexpression of BLT2 has been reported in pancreatic cancer cell lines ${ }^{[16]}$ and BLT2 mediate ERK phosphorylation in pancreatic cancer ${ }^{[19]}$. The activation of BLT2 signaling by LTB4 confers pancreatic cellular proliferation through the MEK/ERK and PI-3 kinase/Akt pathways ${ }^{[21]}$. Together, these observations implicated BLT2 as a novel target for aggressive cancer. However, the correlation between BLT2 expression and its prognostic value in ccRCC remain to be explored.

In the present study, for the first time we used the TCGA (The cancer genome atlas) and GEO (Gene expression omnibus) databases to evaluate the association between BLT2 expression and clinical outcome of ccRCC. In addition, the correlation of BLT2 mRNA levels with the abundance of varied 
immune cells and BLT2-involved molecular mechanisms were investigated. Our findings revealed the potential role of BLT2 in cCRCC and shed light on the underlying mechanism where BLT2 shaped the tumor immune microenvironment, providing a new off-the-shelf therapeutic strategy for fighting ccRCC.

\section{Methods}

\section{Data Source and BLT2 gene expression analysis}

The TIMER database, a bioinformatics initiative including 10,897 samples across 32 cancer types from The Cancer Genome Atlas (TCGA), provides information of cancer transcriptome data to evaluate the potential value of a candidate gene as therapeutic target ${ }^{[22]}$. We analyzed BLT2 differential expression between tumor and normal tissue across different types of cancer using TIMER database with web server (https://cistrome. shinyapps.io/timer/). Furthermore, we investigated the differentially expressed BLT2 mRNA levels between tumor and normal tissues across cancer types using Wilcoxon rank sum test in the integrated datasets combined TCGA with GTEx databases. For the above analysis, RNAseq data in terms of Transcripts per Million (TPM) were collected from UCSC XENA (https://xenabrowser.net/datapages/).

The matched and unmatched differential expression of BLT2 between cancerous and normal tissues for cCRCC was analyzed using paired t-test and Wilcoxon rank sum test, respectively. Gene expression data with clinical information for ccRCC were collected from TCGA (https://portal.gdc.cancer.gov/) in terms of FPKM (Fragment Per Kilobase of transcript per Million mapped read). HTSeq-FPKM data were transformed into TPM for further analyses. Moreover, BLT2 overexpression in kidney cancer on the protein level was identified using immunohistochemistry (IHC) staining data in The Human Protein Atlas (HPA) (https://www.proteinatlas.org/).

\section{Analysis on the clinical value of BLT2 expression}

The correlation between BLT2 expression level and clinicopathologic characteristics were evaluated using a Wilcoxon rank sum test and logistic regression. The distinguishing efficacy of BLT2 expression between normal tissue and ccRCC tumors was estimated using receiver operating characteristic (ROC) curves.

Samples ranking for BLT2 expression above the median were defined as BLT2-high, below the median as BLT2-low. Kaplan-Meier curves were generated to present the association of BLT2 expression with overall survival (OS), progression-free interval (PFI), and disease-specific survival (DSS) for patients with cCRCC from TCGA. Log-rank test was used to establish the statistical significance of the survival curves. The hazard ratio (HR) with 95\% confidence intervals and log-rank p-value were shown in the plots.

Multivariate Cox regression analysis was performed to evaluate the effect of BLT2 expression on OS in the context of other clinical features ( $T$ stage, $\mathrm{N}$ stage, $\mathrm{M}$ stage, pathologic stage, gender and histological grade). The nomogram was constructed using package "rms \& survival" to predict overall survival probability for 1-, 3-, and 5-year based on the six independent prognostic factors. All statistical analyses were performed in R package (V3.6.3). 


\section{Analysis of DEGs between BLT2-high and BLT2-low Expression ccRCC Groups}

Differentially expressed genes (DEGs) were detected between BLT2 ${ }^{\text {high }}$ and BLT2 ${ }^{\text {low }}$ expression ccRCC groups from TCGA datasets using package DESeq2 ${ }^{[23]}$. All the DEmRNAs, DElncRNAs and DEmiRNAs were identified with the adjusted $P$ value $<0.05$ (Student's t-test) and |log fold change [FC]| $>1.0$ defined as the threshold. All the DEGs were presented in volcano plots and the top 15 DEGs were shown in heat maps.

\section{Functional Enrichment Analyses of BLT2-related Genes in ccRCC}

The correlations between BLT2 expression and other genes in cCRCC on the mRNA level were investigated using the $\mathrm{R}$ stats package. Based on Spearman correlation coefficient, the top 30 genes of the strongest positive or negative correlations with BLT2 in cCRCC are shown in heatmaps. The Venn diagram was utilized to perform an intersection analysis to pick out BLT2-correlated DEGs and top 300 BLT2coexpressed genes. Pearson's correlation analyses based on GEPIA database (http://gepia.cancerpku.cn/) were performed to describe the correlations between BLT2 expression and the common genes from the intersection analysis.

Furthermore, the top 300 genes that most positively correlated with BGN were selected to analyze the functional enrichment of BLT2-interacted genes. Gene Ontology (GO) analysis and Kyoto Encyclopedia of Genes and Genomes (KEGG) analysis were performed using the EnrichGO function and EnrichKEGG function in the R package "clusterProfiler" [24].

\section{Correlations between BLT2 expression and tumor immune characteristics in ccRCC}

TIMER2.0 integrates multiple state-of-the-art algorithms for robust estimation of immune infiltration levels for TCGA across diverse cancer types (http://timer.cistrome.org/), including TIMER, xCell, MCPcounter, CIBERSORT, EPIC and quanTIseq ${ }^{[25]}$. TIMER2.0 provides estimation of the relationship between tumor gene expression and immune infiltrates, and allows users to perform gene-gene co-expression pattern study across TCGA cancer types. In the present study, we investigated the association between BLT2 expression and tumor-immune infiltrations, including B cells, CD4+ T cells, CD8+ T cells, neutrophils, macrophages, and myeloid dendritic cells using the 'Gene Module'. In addition, we explored the coexpression pattern of BLT2 with M1 and M2 marker genes and Treg marker genes in 'Gene Corr Module'.

\section{Results}

\section{The Expression Level of BLT2 in ccRCC}

To further evaluate the expression level of BLT2 in human cancers, we used the TIMER database to analyze BLT2 mRNA expression levels in normal tissue and tumors across all TCGA tumor types. Specifically, the result indicated that BLT2 mRNA expression was higher in KIRC (kidney renal clear cell carcinoma) than normal tissues (Figure 1A). Considering the limited samples of tumor-adjacent normal 
tissues in TCGA, we investigated the differential expression of BLT2 mRNA levels between tumor and normal tissues in the integrated datasets combined TCGA with GTEx databases. The analysis revealed that BLT2 was significantly upregulated in KIRC (Figure 1B). The differential expression of BLT2 in CCRCC tumor samples compared with matched and unmatched tumor-adjacent normal tissues was analyzed. The results of the analyses for ccRCC were consistent with the results described above (Figure 1C and Figure 1D). In the end, the differential expression of BLT2 on the protein level between kidney cancer tissues and normal kidney tissues was showed (Figure 1E). Together, the findings identified the overexpression of BLT2 in cCRCC, indicating the potential role of BLT2 during the development and progression of ccRCC.

\section{The clinical value of BLT2 expression in ccRCC}

Subsequently, we investigated the clinical significance of BLT2 in cCRCC. The association of BLT2 with clinicopathological features was shown in Table 1. According to the above results, BLT2 was upregulated in cCRCC. Thus, a ROC curve was constructed to evaluate the distinguishing efficacy of BLT2 between ccRCC tissues and normal kidney tissues. The area under the curve (AUC) of BLT2 is 0.937 (95\% Cl: 0.9150.959), indicative of BLT2 as a potentially valuable identification molecule for ccRCC (Figure 2A). We analyzed the expression of BLT2 in different staging patients with cCRCC, including T staging (T1\&T2 vs T3\&T4), N staging (N0 vs N1\&N2) and M staging (M0 vs M1). The results showed that BLT2 was not differentially expressed in different staging tumors (Figure 2B-2D). Futhermore, the associations between BLT2 expression and clinicopathologic characteristics were investigated using logistic regression. The result was presented in forest plot (Figure 2E), demonstrating that BLT2 expression level was not significantly associated with T staging (T1\&T2 vs T3\&T4), N staging (N0 vs N1\&N2), M staging (M0 vs M1), pathologic stage (Stage $₫ \&$ Stage $\otimes$ vs Stage $\otimes \&$ Stage $\otimes)$ and histologic grade (G1\&G2 vs G3\&G4).

To investigate the prognosis prediction value of BLT2 in patients with CCRCC, we assessed the associations of BLT2 expression levels with the survival for the patients using TCGA database, including OS, DSS and PFI. Notably, Kaplan-Meier survival analysis indicated that high BLT2 expression was significantly correlated with poor OS $(\mathrm{HR}=1.75,95 \% \mathrm{Cl}=1.30-2.36, \mathrm{p} \otimes 0.001)$ and $\mathrm{DSS}(\mathrm{HR}=1.60,95 \% \mathrm{Cl}$ $=1.10-2.34, \mathrm{p} \otimes 0.014$ ) (Figure $3 \mathrm{~A}$ and $3 \mathrm{~B}$ ) for patients with $\mathrm{ccRCC}$, not correlated with PFI (p囚0.303) (Figure 3C).

To quantitatively predict the prognosis of patients with ccRCC, a nomogram was constructed based on multivariate Cox analysis of BLT2 expression and independent clinical risk factors. In the nomogram, a straight line was drawn where a point scale was used to assign points for each predictive variable. The sum of the points from each predictor was assigned to the corresponding location on the total point reference line, where a vertical line was drawn down to the clinical outcome axises. In this way, we find the probability of survival for patients with ccRCC at 1,3, and 5 years. According to the nomogram, the total points of a ccRCC male patient (5 points) with high BLT2 risk (83.75 points), T4 staging (37.5

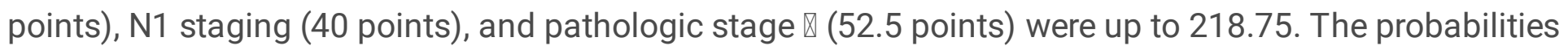
of 1-, 3-, 5-year survival based on the nomogram were about 83.5, 60, and 43.5\% (Figure 3D). 


\section{Identification of DEGs between BLT2-high and BLT2-low ccRCC samples}

Samples of cCRCC from TCGA were sorted into BLT2-high and BLT2-low groups by the median value of BLT2 mRNA expression. In the present study, all the DEIncRNAs, DEmiRNAs and DEmRNAs were identified with the adjusted $P$ value $<0.05$ (Student's t-test) and | log fold change $[F C] \mid>1.0$ as the threshold. $A$ total of 2369 DElncRNAs were identified between the two cohorts, covering 2161 upregulated genes and 208 downregulated genes. In terms of DEmiRNAs, 230 upregulated genes were identified without downregulated genes. As for DEmRNAs, a total of 918 DElncRNAs were identified between the two cohorts, covering 423 upregulated genes and 495 downregulated genes. The distribution of DElncRNAs, DEmiRNAs and DEmRNAs were presented in volcano plots (Figure 4A-4C). The top 15 DEGs (DElncRNAs, DEmiRNAs and DEmRNAs) based on the relative values of expression fold change between the two cohorts were showed in heat maps (Figure 4D-4F).

\section{Identification of BLT2-interacted genes}

To understand relevant molecular functions of BLT2 in cCRCC, the correlations between BLT2 and other genes were evaluated using TCGA data. The top 30 genes that negative and positively correlated with BLT2 expression in ccRCC tumor samples were presented in a heat map, respectively (Figure 5A-5B). Figure 5C showed the intersection of 423 BLT2 expression-sorted DEmRNAs and the top 300 BLT2interacted genes. In the analysis, we screened out the common members such as LTB4R, CCDC17, and NPIPB4. Subsequently, the correlations between BLT2 and the common genes in the intersection were evaluated based on the GEPIA database, and the correlation coefficients were presented as a radar chart (Figure 5D).

\section{Functional Enrichment Analysis of BLT2-interacted genes}

To further explore the potential function and associated pathways of BLT2, the top 300 BLT2-interacted genes were selected for functional enrichment analyses, including GO and KEGG. The results from the GO enrichment analysis showed that BLT2-interactive genes were not significantly involved in pathways associated with biological processes (BPs), cellular compositions (CCs), and molecular functions (MFs) (Figure $6 \mathrm{~A}-6 \mathrm{C}$ ). The results of KEGG pathway enrichment analysis of BLT2-interacted genes were mainly involved in serotonergic synapse, FcERI signaling pathway and arachidonic acid metabolism (Figure 6D).

\section{Correlation of BLT2 expression with immune characteristics in ccRCC}

Tumor-infiltrating lymphocytes (TILs) are an independent predictor of cancer survival and responses to immunotherapy ${ }^{[26,27]}$. Therefore, we evaluated the correlation of BLT2 expression level with the abundance of diverse immune cells in ccRCC based on TIMER2.0, including CD8+ T cells, CD4+T cells, B cells, macrophages, neutrophils, and DCs. BLT2 expression was not significantly correlated with tumor purity in ccRCC. BLT2 expression had significant correlations with the abundance of CD4+ T cells $(r=$ $0.333, P=2.09 e-13)$ and neutrophils $(r=0.258, P=1.81 e-08)$, not with $C D 8+T$ cells, $B$ cells, macrophages, and DCs (Figure 7A). 
In addition, we focused on the correlations between BLT2 and immune marker sets of M1 and M2 macrophages in cCRCC based on TIMER2.0. Notably, we found that M1 marker genes (NOS2 and ROS1) had no significant correlations with BLT2 expression in CCRCC, while moderate correlations were observed between BLT2 expression and M2 marker genes (CD163 and MRC1) (Figure 7B-7C). These findings implied that BLT2 may regulate macrophage M2 polarization in $\mathrm{CCRCC}$, thus contributing to an immunosuppressed microenvironment.

Immunotherapy has gained much attraction due to the widespread use of immune checkpoint inhibitors (ICls) which block tumor-derived immunosuppressed signals, thus amplifying antitumor immunity $[28,29]$. The responses to ICls partly depends on the tumor immune microenvironment such as the abundance of specific immune cells. Subsequently, we investigated the correlations between the expression of BLT2 and that of over 40 common immune-related genes in ccRCC using TCGA data. Notably, the results showed that in CCRCC BLT2 expression was associated with nearly 33 immune checkpoint markers among these genes, including CD274, PDCD1, CTLA4, LAG3, CD160, IDO2 and so on (Figure 8A-8B).

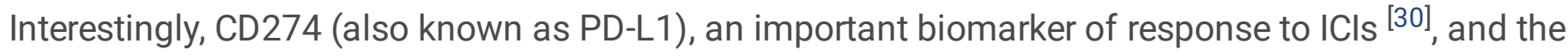
marker genes of T cell exhaustion such as PDCD1, CTLA4, LAG3 ${ }^{[22]}$ positively correlated with BLT2 expression in ccRCC ( $p \bowtie 0.001$ ). Accordingly, these findings implied that BLT2 gene might be involved in the shaping of tumor immunity and $\mathrm{T}$ cell function regulation in ccRCC.

Regulatory $T$ cells (Tregs) maintain immune tolerance and function as a barrier to anti-tumor immunity [31]. We further elucidated the associations between BLT2 expression and immune marker sets of Tregs based on TIMER2.0 and the results showed that BLT2 expression was positively correlated with CCR8 $(r=$ $0.215, p=3.16 e-06)$, Foxp3 $(r=0.167, p=3.11 e-04)$, TGFB1 $(r=0.226, p=9.11 e-07)$ and STAT5B $(r=$ $0.272, p=2.92 e-09$ ) (Figure $8 C$ ). Therefore, these results further suggested that BLT2 played a crucial role in immune escape in the tumor microenvironment of ccRCC. 
Table 1. The associations between BLT2 expression and clinicopathological parameters of ccRCC patients from TCGA-KIRC.

Characteristics No. of patients (n)

p-value test

Low expression of High expression of

LTB4R2 LTB4R2

\section{Gender}

Female

Male

Age

$<=60$

$>60$

T stage

T1

T2

T3

$\mathrm{T} 4$

$\mathrm{N}$ stage

NO

N1

M stage

MO

229

32

137

132

155

33

78

3

113

9

132

138

101

169

0.184

Chi-sq.test

\section{5}

55

123

38

101

8

$0.026 \quad$ Chi-sq.test

Chi-sq.test

M1
128

7

$0.640 \quad$ Chi-sq.test

$0.057 \quad$ Chi-sq.test 199

46

\section{Pathologic} stage

Stage I

151 121

Stage II

28

31

Stage III

56

67

Stage IV

33

49

Histologic grade

G1

5

G2

120

\section{$0.056 \quad$ Chi-sq.test}

31

67

49


G3

G4
104

34
103

41

\section{Discussion}

The main treatment measures for cCRCC are consisted of surgical resection, radiotherapy and chemotherapy, among which ccRCC developed resistance to radiotherapy or conventional chemotherapy [32]. In RCC, surgical resection remains the mainstay of treatment for local tumors. ICls through the blockade of PD1 and CTLA4 has become a new first-line standard option for patients with intermediateor poor-risk metastatic RCC [33]. In 2019, FDA has approved the combined approaches of PD1 inhibitors with anti-angiogenic therapy for advanced RCC [34]. Despite recent advances in ICls, only a minority of patients with RCC will benefit from these therapies, which underscores the need for elucidation of the mechanisms underlying responses to ICls and for search of reliable biomarkers to predict treatment responses. Genomic and transcriptomic changes in the tumors were closely associated with tumor immune infiltrations, thus providing potential targets for improving ICls therapy efficacy.

BLT2, as a chemokine receptor, has been reported to be upregulated in different tumors and associated with tumor cell proliferation and invasion. Considering the limited researches had focused on the role of BLT2 in shaping tumor immune microenvironment, in the present study we performed a comprehensive bioinformatics analysis of BLT2 to unearth its biological functions and tumor-immune interactions in cCRCC. For the first time, we identified the upregulation of BLT2 expression in ccRCC tumors compared with adjacent normal tissues on the mRNA and protein levels. In terms of the prognosis analysis, the results showed that high expression of BLT2 was associated with poorer OS and DSS, indicating the potential value of BLT2 as a prognostic biomarker. Simultaneously, high-expressed BLT2 had excellent distinguishing efficacy between ccRCC tissues and normal kidney tissues based on the high value of AUC (0.937). These findings strongly suggested that BLT2 may act as an oncogene and prognostic biomarker in ccRCC.

To further elucidate the underlying biological function of BLT2 gene in cCRCC, we selected the top 300 genes positively correlated with BLT2 to perform GO and KEGG functional enrichment analysis. The results showed that BLT2-interacted genes were mainly involved in serotonergic synapse, FcERI signaling pathway and arachidonic acid metabolism. To our knowledge, no study has yet assessed the association of above pathways with cancer progression and responses to immunotherapy. In previous reports, it was reported that the activation of BLT2 signaling by LTB4 conferred pancreatic cellular proliferation through the MEK/ERK and PI-3 kinase/Akt pathways ${ }^{[21]}$. During the past 10 years, considerable intratumoural heterogeneity has been reported in $\mathrm{ccRCC}^{[35]}$, which was divided into two distinct subtypes $\mathrm{CcA}$ and $\mathrm{ccB}$ [36]. As previously reported, specific subtypes of $c c R C C$ are associated with responses to sunitinib and share characteristic molecular features such as the expression of PDL1 ${ }^{[37]}$. CcA and ccB tumors differed in their gene expression profiles, and genes associated with hypoxia, angiogenesis, organic acid 
metabolism and fatty acid metabolism were upregulated in ccA tumors ${ }^{[38]}$. According to the findings, BLT2 may be a ccA tumor-related gene. The involved functional pathways and underlying molecular mechanisms of BLT2 during $\mathrm{cCRCC}$ progression deserve further investigations for providing new approaches for targeted therapy in ccRCC.

The abundance of diverse immune cells and the amount of immunomodulatory molecules in tumor microenvironment influences cancer progression and therapeutic treatment responses, especially immunotherapy. In cancer, immunogenicity is the ability to induce adaptive immune responses and cCRCC is considered to be an immunogenic tumour based in part on the clinical benefits for patients from IL-2 and interferon- a (IFNa) treatments ${ }^{[39]}$. These clinical observations suggested that promoting endogenous anti-tumour immunity could be an effective therapeutic strategy for ccRCC. In the present study, we determined whether BLT2 expression level could influence the abundance and function of various immune cells in $\mathrm{CCRCC}$, which in turn, favors tumor progression and promotes an immunosuppressive microenvironment. Our results demonstrate that there was a moderate positive relationships between BLT2 expression level and infiltration level of neutrophils, which is in line with its biological function as the receptor of LTB4, a potent neutrophil chemoattractant. Moreover, our results uncover a crucial role of BLT2 in regulating tumor immunity in ccRCC through correlation analyses of BLT2 expression and the marker genes of immune cells (macrophages and T cells). First, M1 marker genes (NOS2 and ROS1) had no significant correlations with BLT2 expression in ccRCC, while moderate correlations were observed between BLT2 expression and M2 marker genes (CD163 and MRC1). The finding revealed the potential regulating role of BLT2 in polarization of tumor-associated macrophages (TAM). In addition, our results revealed that BLT2 had the potential to activate Tregs and promote T cell exhaustion in CCRCC. Increased expression of BLT2 positively correlated with the marker gene expression of Treg and T cell exhaustion (FOXP3, CCR8, STAT5B, TGFB1, PD-1, CTLA4, and LAG3). These findings implied that increased expression of BLT2 may contribute to an immunosuppressed microenvironment in ccRCC.

In summary, the findings of our study uncovered that increased BLT2 expression correlates with poor prognosis. In addition, BLT2 expression potentially contributes to the regulation of TAMs, T cell exhaustion, and Tregs activation in ccRCC. Therefore, BLT2 may serve as a novel prognostic biomarker and shape tumor immune microenvironment in ccRCC. The involved functional pathways and underlying molecular mechanisms of BLT2 during ccRCC progression deserve further investigations for providing new approaches to drive the development of new immunotherapeutic strategies for ccRCC.

\section{Declarations}

\section{Funding}

This study was supported by Hunan Cancer Hospital Climb Plan (No. 2020NSFC-B005), Scientific Research Project of Hunan Provincial Health Commission (B2013-097), and Science and Technology Innovation Program of Hunan Province (2018SK50907). 


\section{Conflicts of interest}

The authors confirm that there are no conflicts of interest.

\section{Availability of data and material}

We acknowledge the TIMER, TCGA, GTEx, GEPIA, and HPA databases for free use. All data is available under reasonable request.

\section{Code availability}

Not applicable.

\section{Author Contribution Statement}

W.W. and Y.H. provided the idea and designed the article. X.Y. analyzed the data and wrote the manuscript. X.Y. and C.L. edited the figures. W.W. and Y.H. reviewed the manuscript.

\section{Ethics approval}

This article is not involved in any studies with human participants or animals.

\section{Consent to participate}

Not applicable.

\section{Consent for publication}

All authors consent to the publication of this study.

\section{Declarations}

1. Li YL, Wu LW, Zeng LH, Zhang ZY, Wang W. ApoC1 promotes the metastasis of clear cell renal cell carcinoma via activation of STAT3. 2020, 39(39): 6203-6217.

2. Minton DR, Fu L, Mongan NP, Shevchuk MM, Nanus DM, Gudas LJ. Role of NADH Dehydrogenase (Ubiquinone) 1 Alpha Subcomplex 4-Like 2 in Clear Cell Renal Cell Carcinoma. Clin Cancer Res 2016, 22(11): 2791-2801.

3. Mitchell TJ, Turajlic S, Rowan A, Nicol D, Farmery JHR, O'Brien T, Martincorena I, Tarpey P, Angelopoulos N, Yates LR, Butler AP, Raine K, Stewart GD, Challacombe B, Fernando A, Lopez JI, Hazell S, Chandra A, Chowdhury S, Rudman S, Soultati A, Stamp G, Fotiadis N, Pickering L, Au L, Spain L, Lynch J, Stares M, Teague J, Maura F, Wedge DC, Horswell S, Chambers T, Litchfield K, Xu H, Stewart A, Elaidi R, Oudard S, McGranahan N, Csabai I, Gore M, Futreal PA, Larkin J, Lynch AG, Szallasi Z, Swanton C, 
Campbell PJ. Timing the Landmark Events in the Evolution of Clear Cell Renal Cell Cancer: TRACERx Renal. Cell 2018, 173(3): 611-623.e617.

4. Schödel J, Grampp S, Maher ER, Moch H, Ratcliffe PJ, Russo P, Mole DR. Hypoxia, Hypoxiainducible Transcription Factors, and Renal Cancer. European urology 2016, 69(4): 646-657.

5. Chen M, Zhang S, Nie Z, Wen X, Gao Y. Identification of an Autophagy-Related Prognostic Signature for Clear Cell Renal Cell Carcinoma. Frontiers in oncology 2020, 10: 873-873.

6. Ochocki JD, Khare S, Hess M, Ackerman D, Qiu B, Daisak JI, Worth AJ, Lin N, Lee P, Xie H, Li B, Wubbenhorst B, Maguire TG, Nathanson KL, Alwine JC, Blair IA, Nissim I, Keith B, Simon MC. Arginase 2 Suppresses Renal Carcinoma Progression via Biosynthetic Cofactor Pyridoxal Phosphate Depletion and Increased Polyamine Toxicity. Cell metabolism 2018, 27(6): 1263-1280.e1266.

7. Reustle A, Di Marco M, Meyerhoff C, Nelde A, Walz JS, Winter S, Kandabarau S, Büttner F, Haag M, Backert L, Kowalewski DJ, Rausch S, Hennenlotter J, Stühler V, Scharpf M, Fend F, Stenzl A, Rammensee H-G, Bedke J, Stevanović S, Schwab M, Schaeffeler E. Integrative -omics and HLA-ligandomics analysis to identify novel drug targets for ccRCC immunotherapy. Genome Med 2020, 12(1): 32-32.

8. Courtney KD, Infante JR, Lam ET, Figlin RA, Rini BI, Brugarolas J, Zojwalla NJ, Lowe AM, Wang K, Wallace EM, Josey JA, Choueiri TK. Phase I Dose-Escalation Trial of PT2385, a First-in-Class HypoxiaInducible Factor-2a Antagonist in Patients With Previously Treated Advanced Clear Cell Renal Cell Carcinoma. Journal of clinical oncology : official journal of the American Society of Clinical Oncology 2018, 36(9): 867-874.

9. Ascierto PA, Brugarolas J, Buonaguro L, Butterfield LH, Carbone D, Daniele B, Ferris R, Fox BA, Galon J, Gridelli C, Kaufman HL, Klebanoff CA, Melero I, Nathan P, Paulos CM, Ruella M, Sullivan R, Zarour $\mathrm{H}$, Puzanov I. Perspectives in immunotherapy: meeting report from the Immunotherapy Bridge (29-30 November, 2017, Naples, Italy). Journal for immunotherapy of cancer 2018, 6(1): 69-69.

10. Liu Z, Liu Y, Xu L, An H, Chang Y, Yang Y, Zhang W, Xu J. P2X7 receptor predicts postoperative cancer-specific survival of patients with clear-cell renal cell carcinoma. Cancer science 2015, 106(9): 1224-1231.

11. Montemagno C, Hagege A, Borchiellini D, Thamphya B, Rastoin O, Ambrosetti D, lovanna J, RiouxLeclercq N, Porta C, Negrier S, Ferrero J-M, Chamorey E, Pagès G, Dufies M. Soluble forms of PD-L1 and PD-1 as prognostic and predictive markers of sunitinib efficacy in patients with metastatic clear cell renal cell carcinoma. Oncoimmunology 2020, 9(1): 1846901-1846901.

12. Vuong L, Kotecha RR, Voss MH, Hakimi AA. Tumor Microenvironment Dynamics in Clear-Cell Renal Cell Carcinoma. Cancer discovery 2019, 9(10): 1349-1357. 
13. Satpathy SR, Jala VR, Bodduluri SR, Krishnan E, Hegde B, Hoyle GW, Fraig M, Luster AD, Haribabu B. Crystalline silica-induced leukotriene B4-dependent inflammation promotes lung tumour growth. Nat Commun 2015, 6: 7064.

14. Steinhilber D, Fischer AS, Metzner J, Steinbrink SD, Roos J, Ruthardt M, Maier TJ. 5-lipoxygenase: underappreciated role of a pro-inflammatory enzyme in tumorigenesis. Frontiers in pharmacology 2010, 1: 143 .

15. He R, Chen Y, Cai Q. The role of the LTB4-BLT1 axis in health and disease. Pharmacological research 2020, 158: 104857.

16. Hennig R, Osman T, Esposito I, Giese N, Rao SM, Ding XZ, Tong WG, Büchler MW, Yokomizo T, Friess H, Adrian TE. BLT2 is expressed in PanINs, IPMNs, pancreatic cancer and stimulates tumour cell proliferation. Br J Cancer 2008, 99(7): 1064-1073.

17. Metzemaekers M, Gouwy M, Proost P. Neutrophil chemoattractant receptors in health and disease: double-edged swords. Cellular \& molecular immunology 2020, 17(5): 433-450.

18. Bäck M, Powell WS, Dahlén S-E, Drazen JM, Evans JF, Serhan CN, Shimizu T, Yokomizo T, Rovati GE. Update on leukotriene, lipoxin and oxoeicosanoid receptors: IUPHAR Review 7. Br J Pharmacol 2014, 171(15): 3551-3574.

19. Cho N-K, Joo Y-C, Wei JD, Park JI, Kim J-H. BLT2 is a pro-tumorigenic mediator during cancer progression and a therapeutic target for anti-cancer drug development. American journal of cancer research 2013, 3(4): 347-355.

20. Kim H, Park GS, Lee JE, Kim JH. A leukotriene B4 receptor-2 is associated with paclitaxel resistance in MCF-7/DOX breast cancer cells. Br J Cancer 2013, 109(2): 351-359.

21. Azrad M, Turgeon C, Demark-Wahnefried W. Current evidence linking polyunsaturated Fatty acids with cancer risk and progression. Frontiers in oncology 2013, 3: 224-224.

22. Pan J-h, Zhou H, Cooper L, Huang J-I, Zhu S-b, Zhao X-X, Ding H, Pan Y-I, Rong L. LAYN Is a Prognostic Biomarker and Correlated With Immune Infiltrates in Gastric and Colon Cancers. Frontiers in immunology 2019, 10(6).

23. Love MI, Huber W, Anders S. Moderated estimation of fold change and dispersion for RNA-seq data with DESeq2. Genome biology 2014, 15(12): 550.

24. Yu G, Wang LG, Han Y, He QY. clusterProfiler: an R package for comparing biological themes among gene clusters. Omics : a journal of integrative biology 2012, 16(5): 284-287.

25. Li T, Fu J, Zeng Z, Cohen D, Li J, Chen Q, Li B, Liu XS. TIMER2.0 for analysis of tumor-infiltrating immune cells. Nucleic acids research 2020, 48(W1): W509-w514. 
26. Ohtani H. Focus on TILs: prognostic significance of tumor infiltrating lymphocytes in human colorectal cancer. Cancer immunity 2007, 7: 4.

27. Yang RK, Kuznetsov IB, Ranheim EA, Wei JS, Sindiri S, Gryder BE, Gangalapudi V, Song YK, Patel V, Hank JA, Zuleger C, Erbe AK, Morris ZS, Quale R, Kim K, Albertini MR, Khan J, Sondel PM. OutcomeRelated Signatures Identified by Whole Transcriptome Sequencing of Resectable Stage III/IV Melanoma Evaluated after Starting Hu14.18-IL2. Clin Cancer Res 2020, 26(13): 3296-3306.

28. Topalian SL, Drake CG, Pardoll DM. Immune checkpoint blockade: a common denominator approach to cancer therapy. Cancer Cell 2015, 27(4): 450-461.

29. Zhou X, Zhu X, Yao J, Wang X, Wang N. Comprehensive analysis of clinical prognosis and molecular immune characterization of tropomyosin 4 in pancreatic cancer. 2021.

30. Doroshow DB, Bhalla S, Beasley MB, Sholl LM, Kerr KM, Gnjatic S. PD-L1 as a biomarker of response to immune-checkpoint inhibitors. 2021, 18(6): 345-362.

31. Cervantes-Villagrana RD, Albores-García D, Cervantes-Villagrana AR, García-Acevez SJ. Tumorinduced neurogenesis and immune evasion as targets of innovative anti-cancer therapies. Signal transduction and targeted therapy 2020, 5(1): 99-99.

32. Mu Z, Dong D, Sun M, Li L, Wei N, Hu B. Prognostic Value of YTHDF2 in Clear Cell Renal Cell Carcinoma. Frontiers in oncology 2020, 10:1566-1566.

33. Amin A, Hammers H. The Evolving Landscape of Immunotherapy-Based Combinations for Frontline Treatment of Advanced Renal Cell Carcinoma. Frontiers in immunology 2018, 9: 3120.

34. Escudier B. Combination Therapy as First-Line Treatment in Metastatic Renal-Cell Carcinoma. N Engl J Med 2019, 380(12): 1176-1178.

35. Gerlinger M, Horswell S, Larkin J, Rowan AJ, Salm MP, Varela I, Fisher R, McGranahan N, Matthews N, Santos CR, Martinez P, Phillimore B, Begum S, Rabinowitz A, Spencer-Dene B, Gulati S, Bates PA, Stamp G, Pickering L, Gore M, Nicol DL, Hazell S, Futreal PA, Stewart A, Swanton C. Genomic architecture and evolution of clear cell renal cell carcinomas defined by multiregion sequencing. Nature genetics 2014, 46(3): 225-233.

36. Brannon AR, Reddy A, Seiler M, Arreola A, Moore DT, Pruthi RS, Wallen EM, Nielsen ME, Liu H, Nathanson KL, Ljungberg B, Zhao H, Brooks JD, Ganesan S, Bhanot G, Rathmell WK. Molecular Stratification of Clear Cell Renal Cell Carcinoma by Consensus Clustering Reveals Distinct Subtypes and Survival Patterns. Genes \& cancer 2010, 1(2): 152-163.

37. Beuselinck B, Verbiest A, Couchy G, Job S, de Reynies A, Meiller C, Albersen M, Verkarre V, Lerut E, Méjean A, Patard JJ, Laguerre B, Rioux-Leclercq N, Schöffski P, Oudard S, Zucman-Rossi J. Pro- 
angiogenic gene expression is associated with better outcome on sunitinib in metastatic clear-cell renal cell carcinoma. Acta oncologica (Stockholm, Sweden) 2018, 57(4): 498-508.

38. Beuselinck B, Job S, Becht E, Karadimou A, Verkarre V, Couchy G, Giraldo N, Rioux-Leclercq N, Molinié V, Sibony M, Elaidi R, Teghom C, Patard JJ, Méjean A, Fridman WH, Sautès-Fridman C, de Reyniès A, Oudard S, Zucman-Rossi J. Molecular subtypes of clear cell renal cell carcinoma are associated with sunitinib response in the metastatic setting. Clin Cancer Res 2015, 21(6):1329-1339.

39. Kirkwood JM, Ernstoff MS. Interferons in the treatment of human cancer. Journal of clinical oncology : official journal of the American Society of Clinical Oncology 1984, 2(4):336-352.

\section{Figures}

A

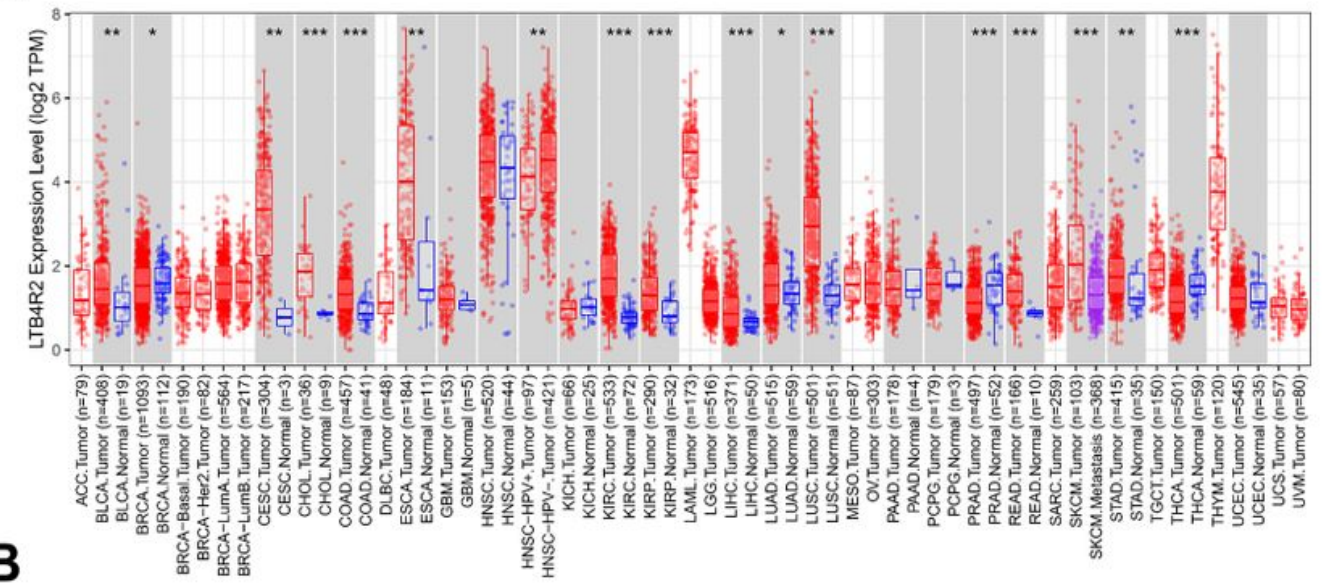

C
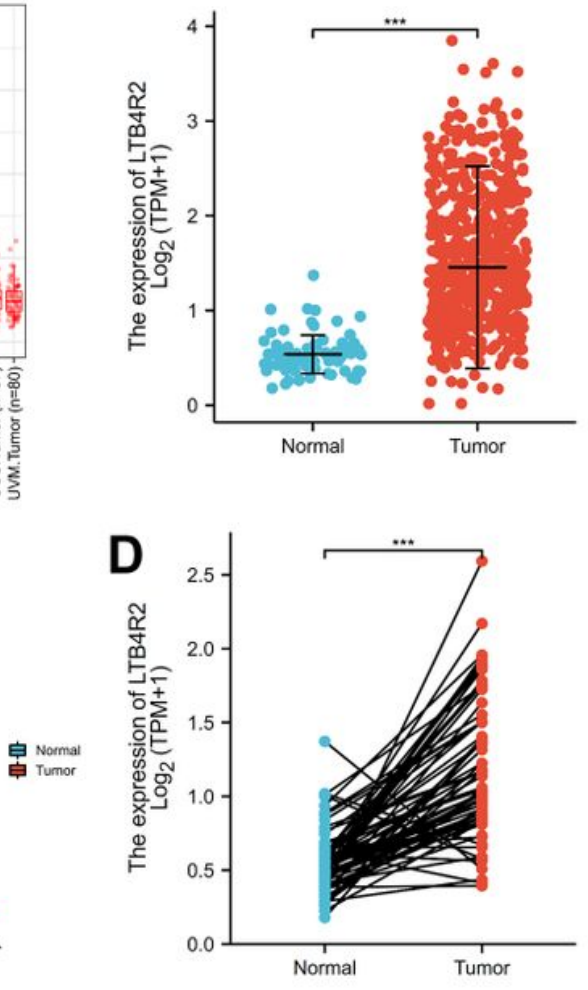

E

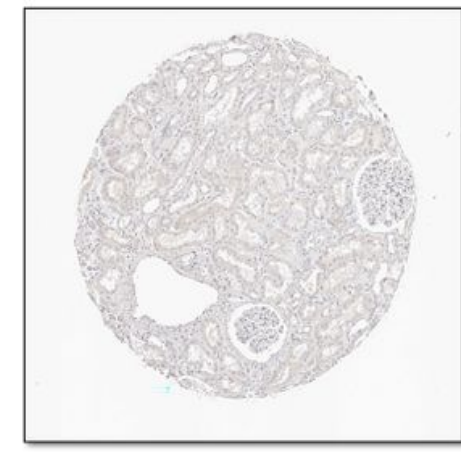

Normal kidney

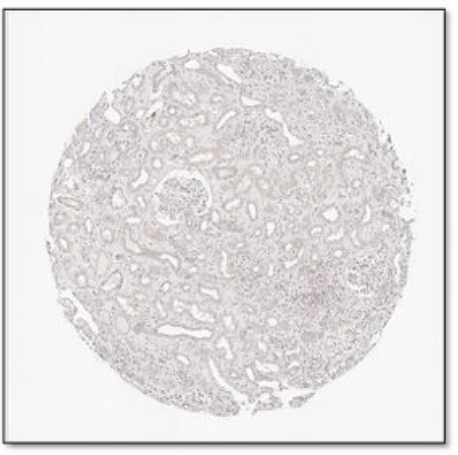

Kidney cancer

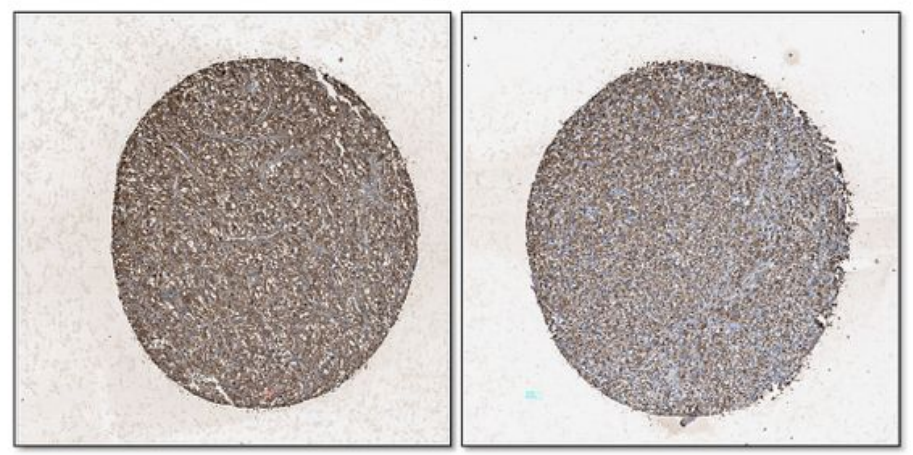


Figure 1

The differential expression levels of BLT2 in cCRCC. (A) BLT2 expression in cancerous and normal tissues across different cancers based on TIMER. (B) BLT2 expression in cancerous and normal tissues across different cancers from TCGA and GTEx pan-cancer data. (C) BLT2 expression in ccRCC and normal tissues from TCGA. (D) BLT2 expression in CCRCC and matched tumor-adjacent normal tissues from TCGA. (E) BLT2 expression (immunohistochemistry) in cCRCC on the protein level from the Human

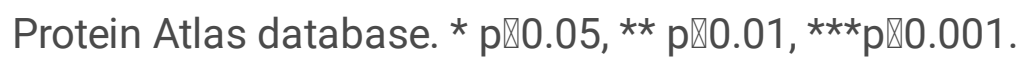
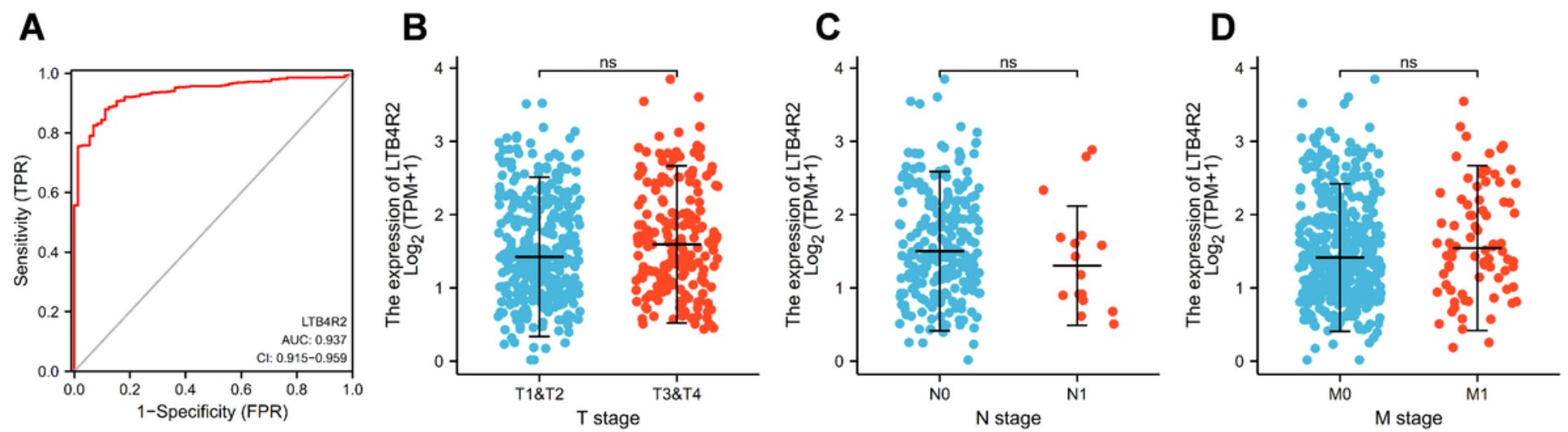

E

\begin{tabular}{|c|c|c|c|c|}
\hline Characteristics & Total $(\mathrm{N})$ & $\mathrm{OR}(95 \% \mathrm{Cl})$ & & $P$ value \\
\hline T stage (T3\&T4 vs. T1\&T2) & 539 & $1.290(0.906-1.841)$ & $\frac{1}{1}-1$ & 0.159 \\
\hline $\mathrm{N}$ stage (N1 vs. N0) & 257 & $0.734(0.255-2.032$ & 1 & 0.552 \\
\hline M stage (M1 vs. M0) & 506 & $1.293(0.798-2.106)$ & $\frac{1}{1}$ & 0.298 \\
\hline Pathologic stage (Stage III\&Stage IV vs. Stage I\&Stage II) & 536 & $1.268(0.895-1.799)$ & $\underset{1}{1}+$ & 0.183 \\
\hline Histologic grade (G3\&G4 vs. G1\&G2) & 531 & $0.891(0.634-1.254)$ & $\vdash \frac{1}{1}$ & 0.509 \\
\hline
\end{tabular}

\section{Figure 2}

Correlations between BLT2 expression and clinicopathological features. (A) A ROC curve was generated to evaluate the distinguishing efficacy of BLT2 mRNA expression between ccRCC and normal kidney tissues. (B) Association of BLT2 expression with T stage. (C) Association of BLT2 expression with N stage. (D) Association of BLT2 expression with M stage. (E) The correlation between BLT2 expression level and clinicopathologic characteristics evaluated using a logistic regression. 
A

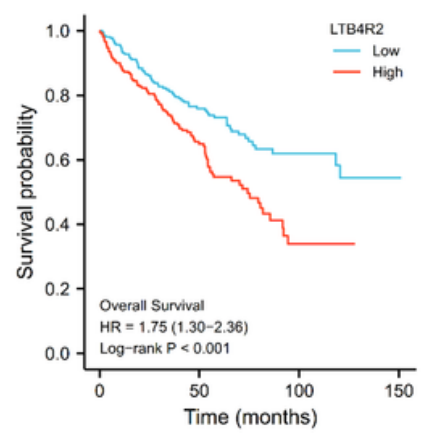

B

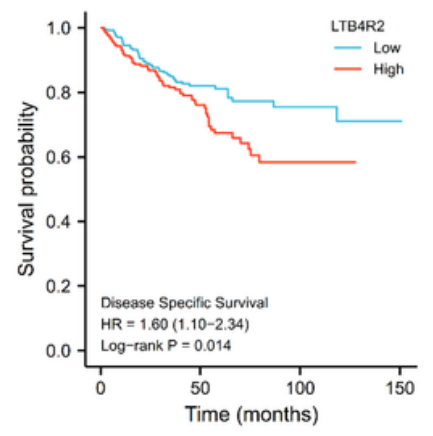

C

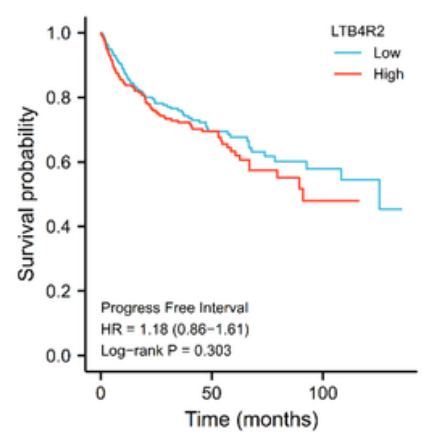

Points

T stage

$\mathrm{N}$ stage

M stage

Pathologic stage

Gender

Histologic grade

LTB4R2

Total Points

Linear Predictor

1-year Survival Probability

3-year Survival Probability

5-year Survival Probability
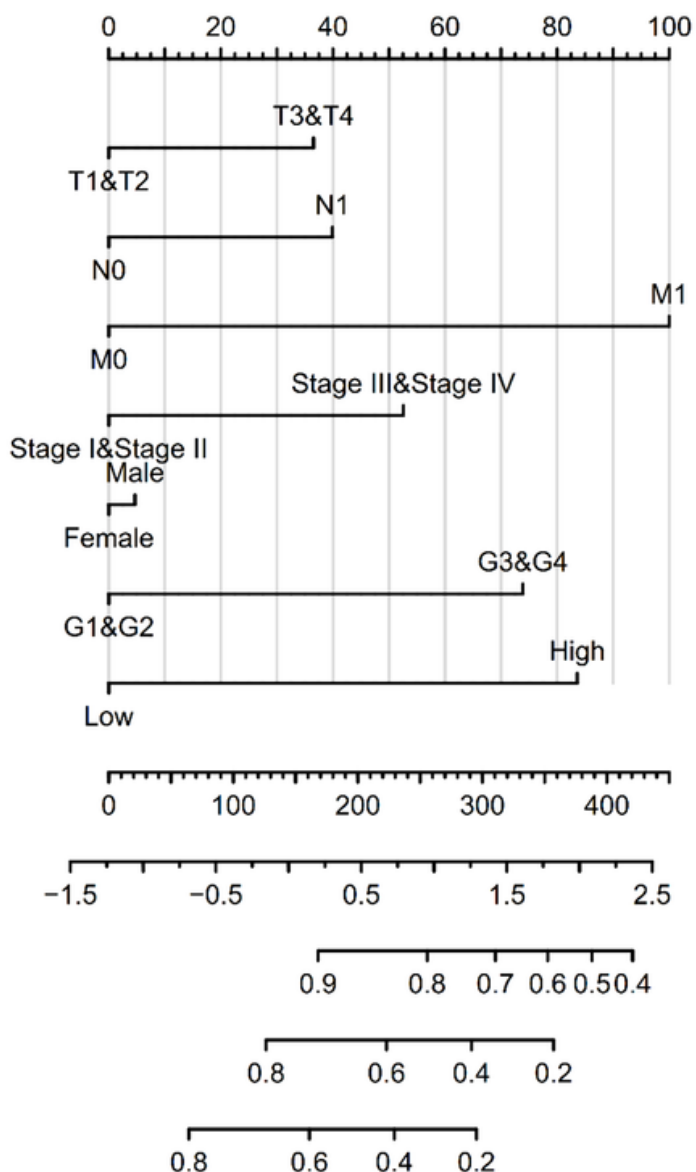

\section{Figure 3}

The prognostic value of BLT2 mRNA expression for patients with ccRCC. (A) Kaplan-Meier survival curves of overall survival between BLT2-high and -low patients with ccRCC. (B) Kaplan-Meier survival curves of disease specific survival between BLT2-high and -low patients with cCRCC. (C) Kaplan-Meier survival curves of progression free interval between BLT2-high and -low patients with ccRCC. (D) The nomogram for predicting overall survival probability of $1-, 3$-, and 5 -year based on multivariate Cox regression

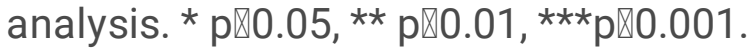


A

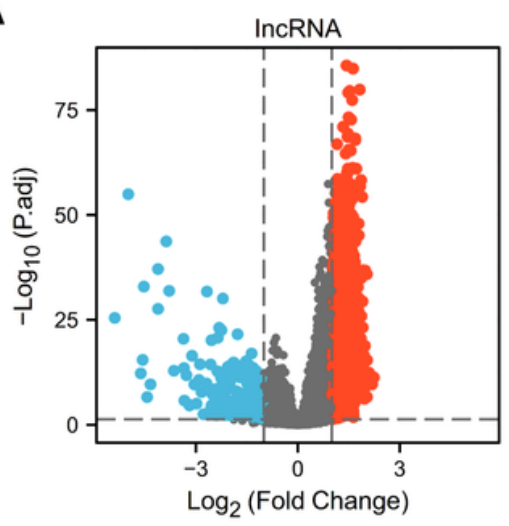

D

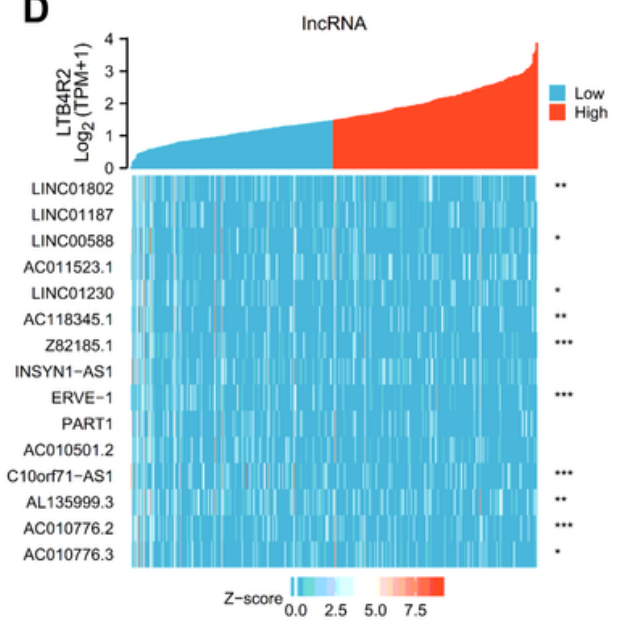

B

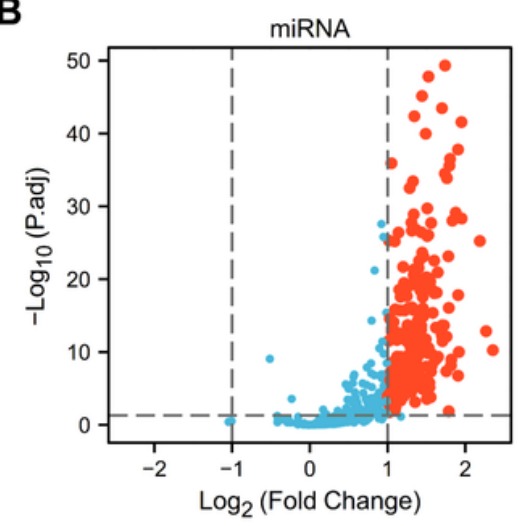

E

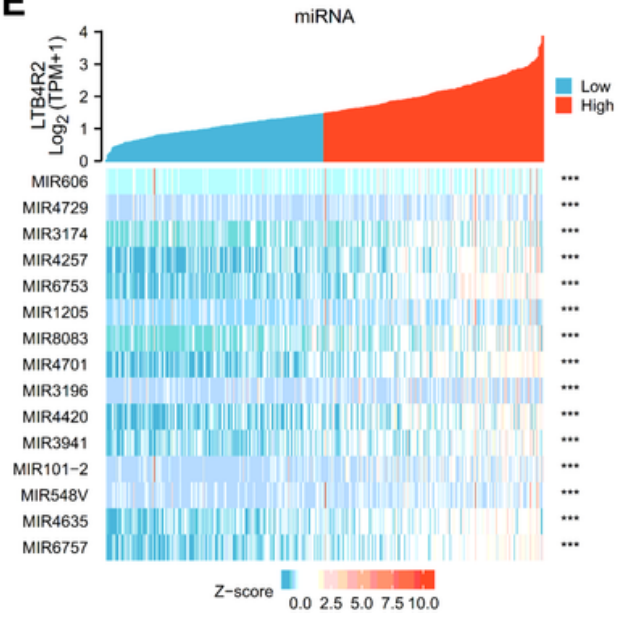

C

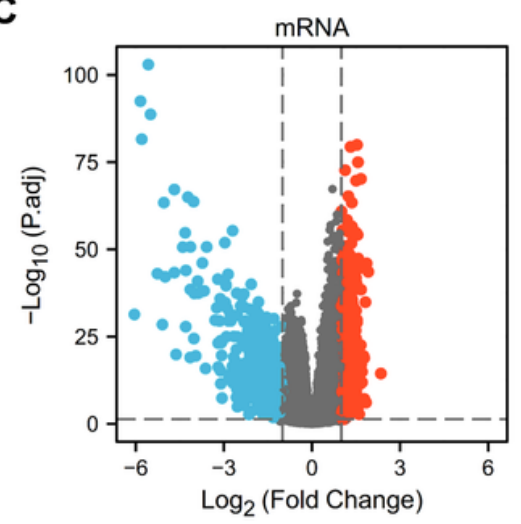

$\mathbf{F}$

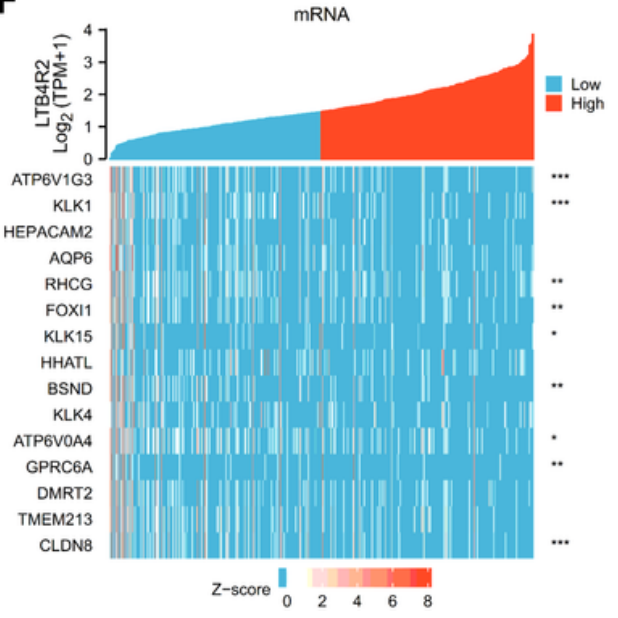

Figure 4

DEGs between the BLT2high and BLT2low expression groups in ccRCC samples. (A) Volcano plot of DElncRNAs. (B) Volcano plot of DEmiRNAs. (C) Volcano plot of DEmRNAs. (D) Heat map of the top 15 DElncRNAs. (E) Heat map of the top 15 DEmiRNAs. (F) Heat map of the top 15 DEmRNAs. 
A

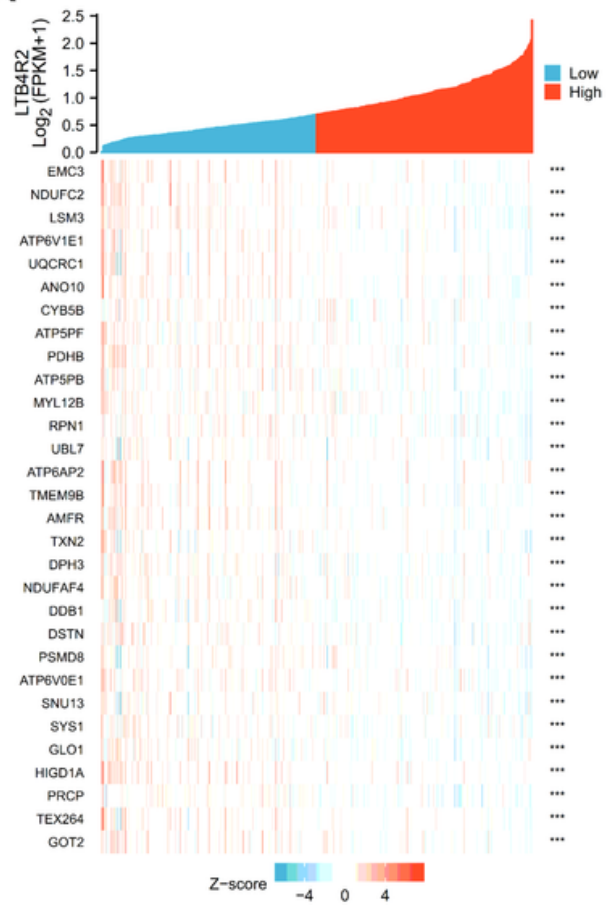

B

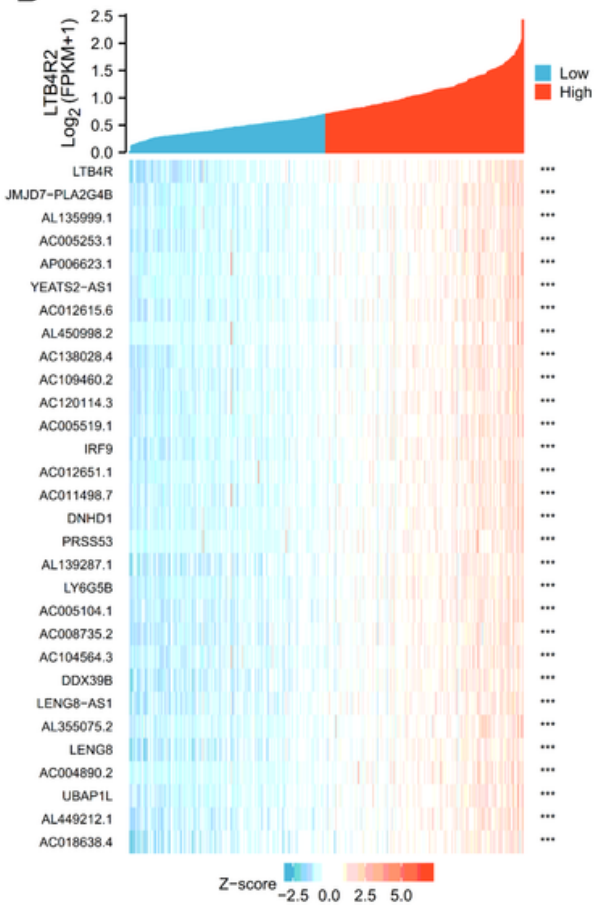

C

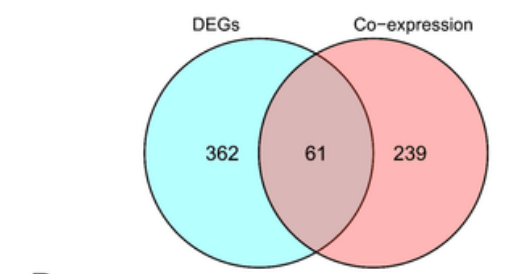

D

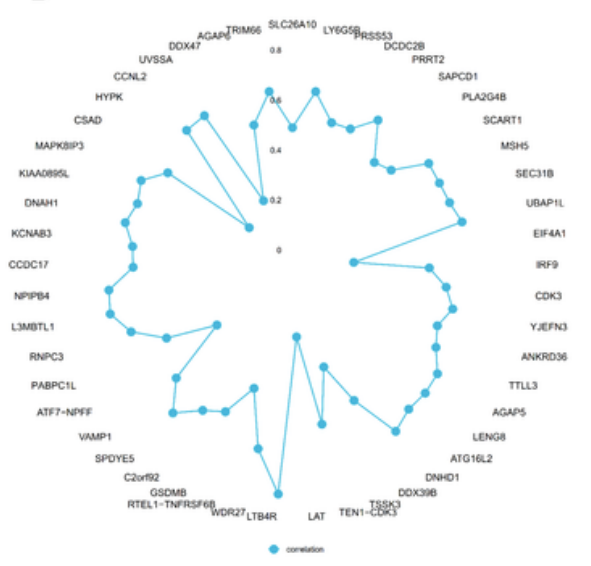

Figure 5

BLT2-related genes in ccRCC. (A) Heat map of the top 30 genes most negatively associated with BLT2 in ccRCC. (B) Heat map of the top 30 genes most positively associated with BLT2 in ccRCC. (C) The Venn diagram of an intersection analysis to pick out BLT2-correlated DEGs and top 300 BLT2-coexpressed genes. (D) The radar chart of the correlations between BLT2 and the common genes in the intersection based on the GEPIA database.
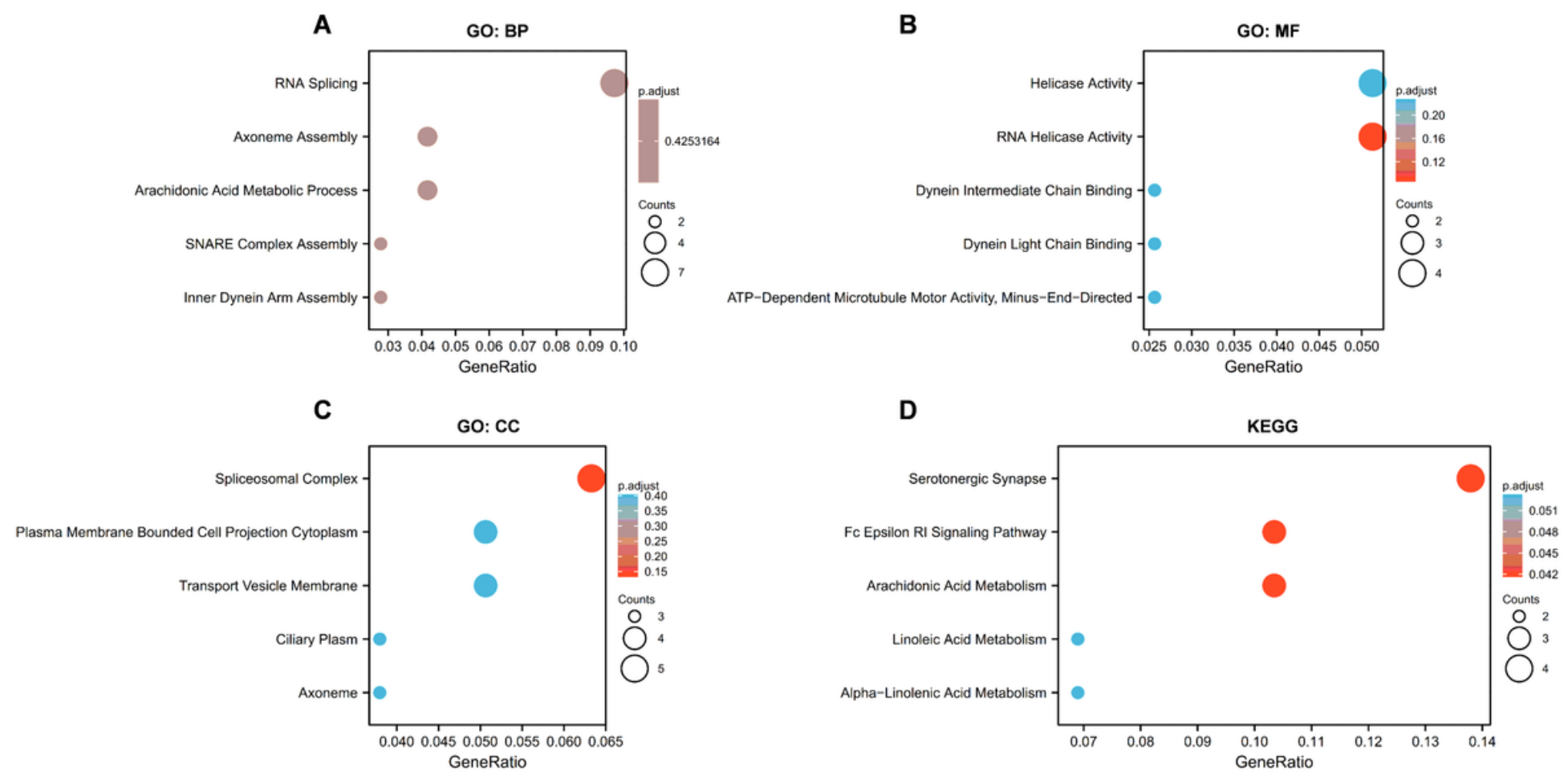

Figure 6 
Function and pathway enrichment analyses of BLT2-related genes in ccRCC. (A-C) GO analysis of the top 300 BLT2-related genes, including biological processes (BP), molecular function (MF), and cell component (CC). (D) KEGG pathway analysis of the top 300 BLT2-related genes.

A
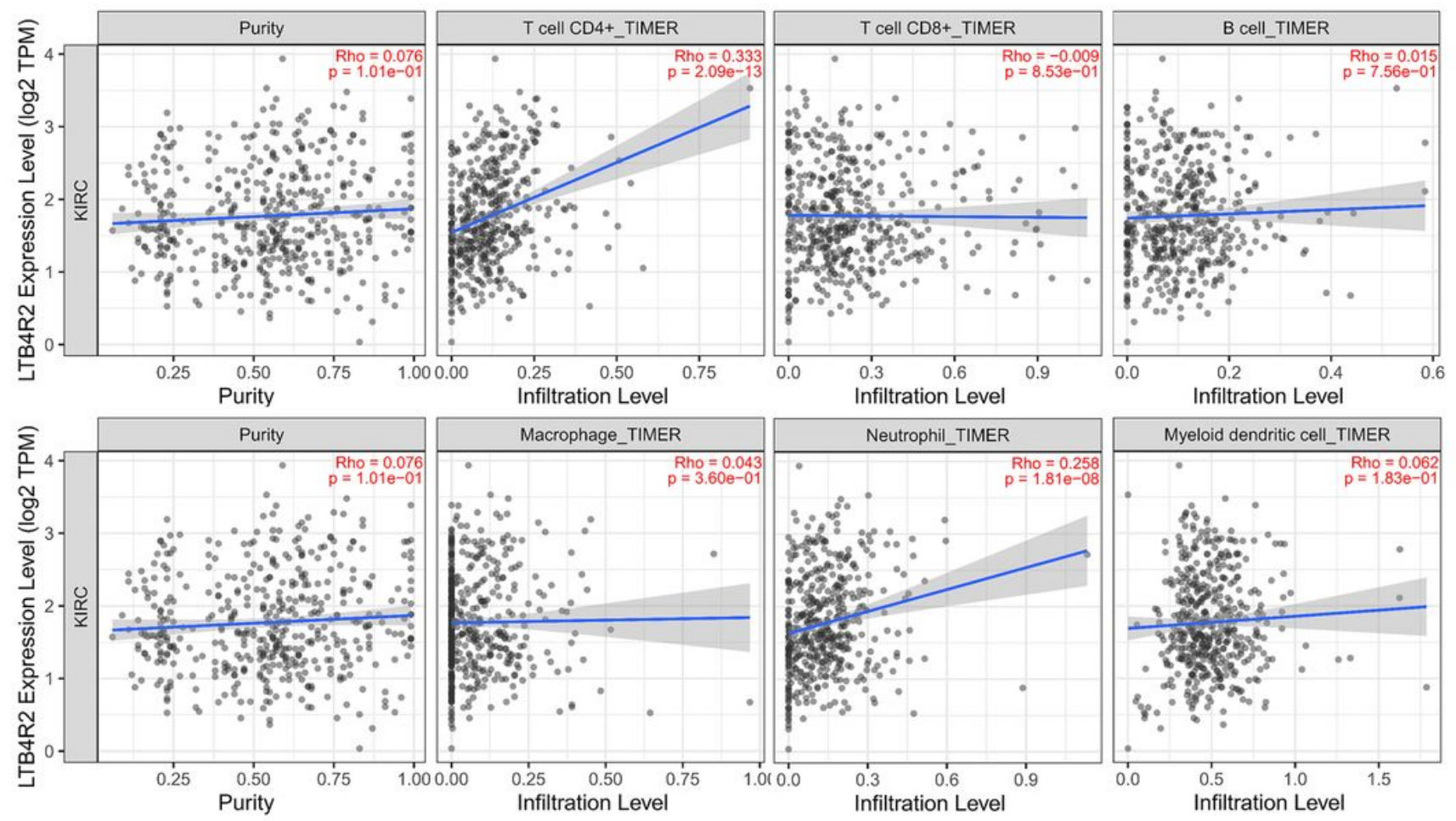

B
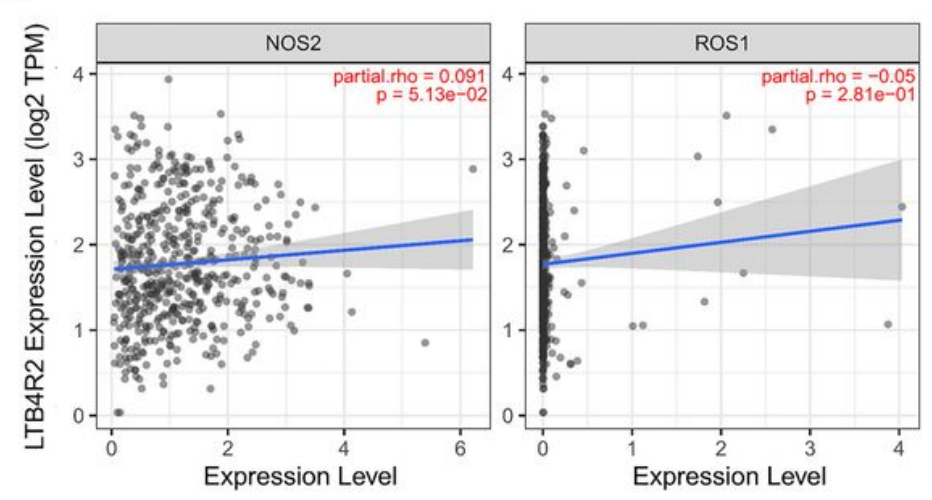

C

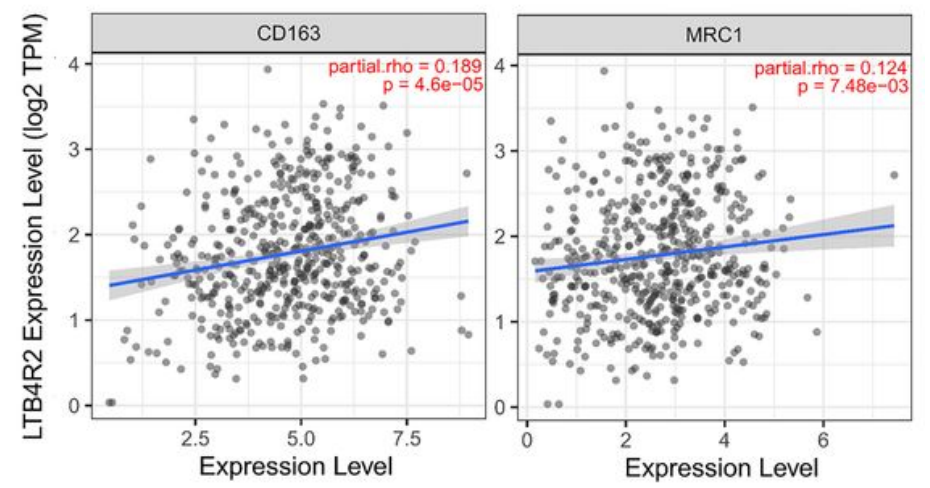

Figure 7

Correlations between BLT2 expression and immune cell infiltration levels in CCRCC. (A) Correlation between BLT2 expression level and the abundance of 6 immune cells in ccRCC based on TIMER2.0. (B) Correlations between BLT2 expression and immune marker genes of M1 macrophages in cCRCC based on TIMER2.0. (C) Correlations between BLT2 expression and immune marker genes of M2 macrophages in cCRCC based on TIMER2.0. 


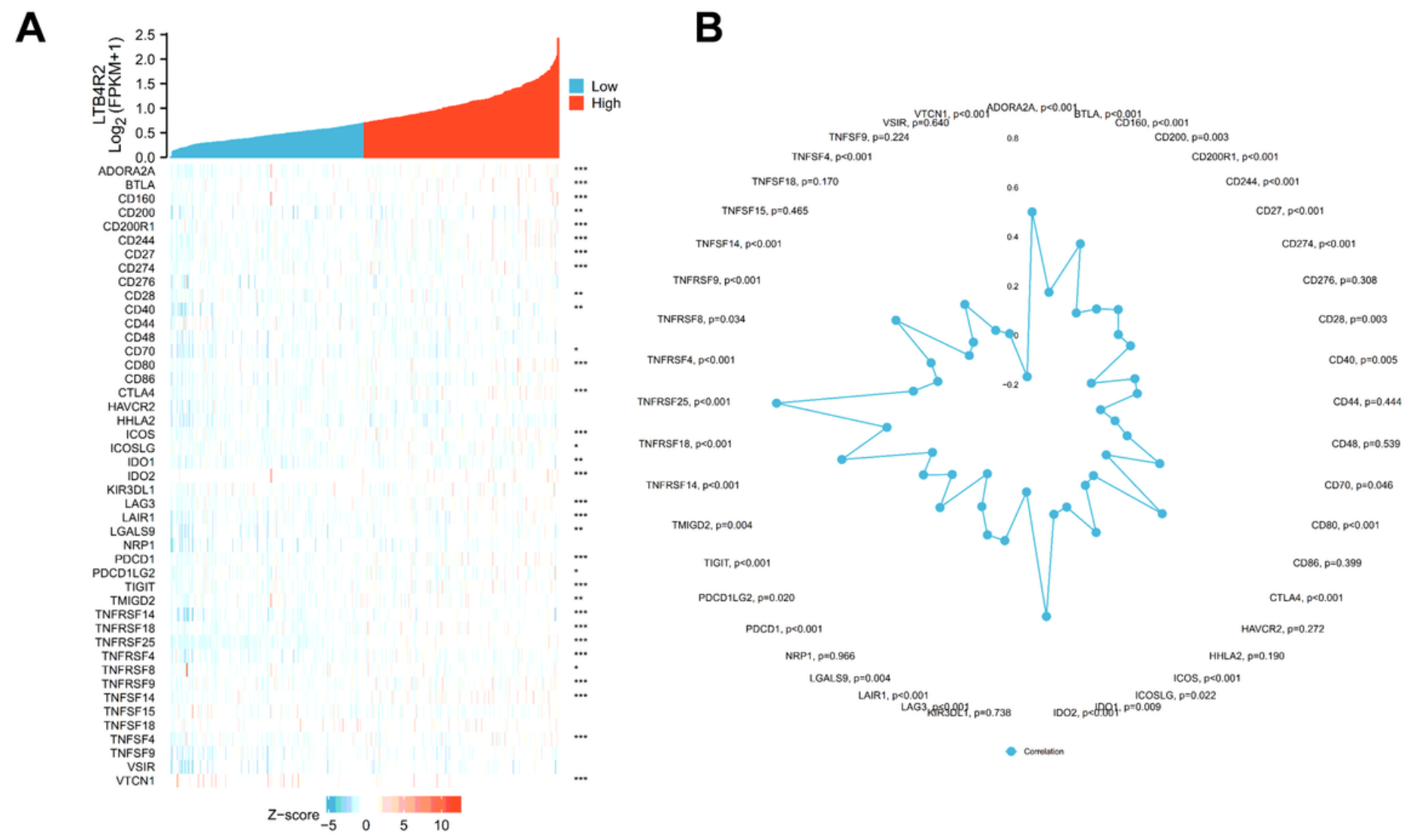

C
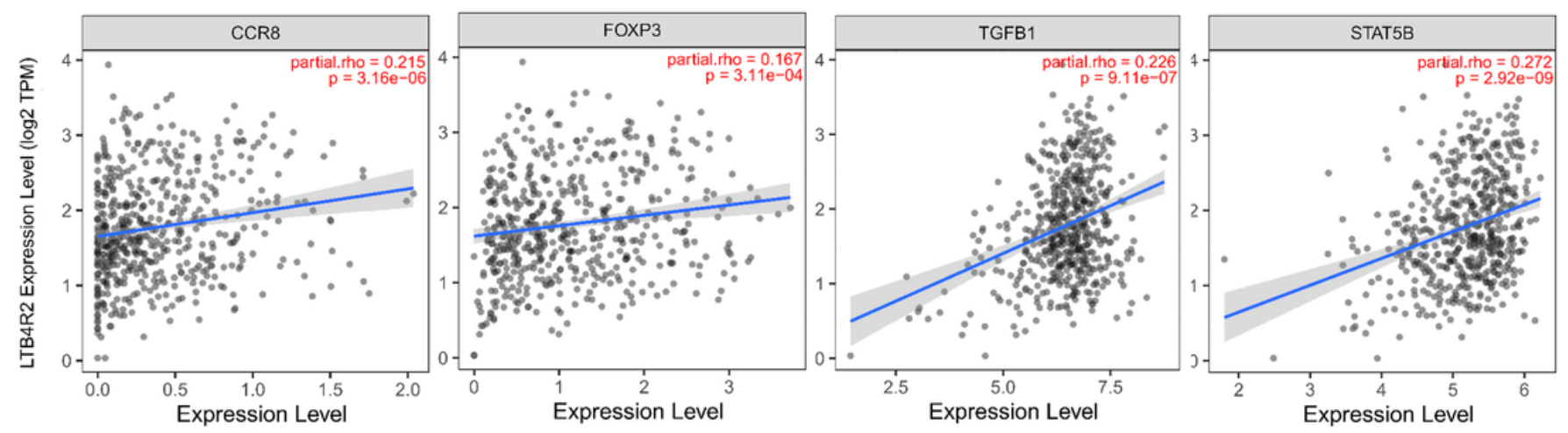

Figure 8

Correlations between BLT2 expression level with immune checkpoint gene levels. (A) Heat map of the correlations between BLT2 expression with over 40 common immune checkpoint gene in ccRCC. (B) The radar chart of the correlations between BLT2 expression with over 40 common immune checkpoint genes in cCRCC. (C) Correlations between BLT2 expression and immune marker genes of Tregs in ccRCC based on TIMER database. 